Ciência Florestal, Santa Maria, v. 22, n. 3, p. 467-476, jul.-set., 2012 ISSN 0103-9954

\title{
PARTIÇÃO DE BIOMASSA SECA E NUTRIENTE EM MINICEPAS DE EUCALIPTO INFLUENCIADA PELA ADUBAÇÃO NPK
}

\author{
PARTITION OF DRY BIOMASS AND NUTRIENT IN MINISTUMPS OF Eucalyptus \\ INFLUENCED BY NPK FERTILIZATION
}

\author{
José Pereira Carvalho Neto ${ }^{1}$ Enilson de Barros Silva ${ }^{2}$ Reynaldo Campos Santana ${ }^{3}$ \\ Paulo Henrique Grazziotti ${ }^{4}$ José Sebastião Cunha Fernandes ${ }^{5}$ \\ Juan Paulo Xavier de Freitas ${ }^{6}$ Cássio Vinicius de Souza ${ }^{6}$
}

\begin{abstract}
RESUMO
O estado nutricional da planta-matriz é de grande importância para a manutenção do seu vigor vegetativo, o que determina a produção de brotos e também os índices de enraizamento e velocidade de formação das raízes, uma vez que os macronutrientes e os micronutrientes estão envolvidos nos processos bioquímicos e fisiológicos vitais à planta. Nesse sentido, o objetivo deste trabalho foi avaliar a influência da adubação NPK sobre a partição de biomassa seca e nutriente em minicepa de eucalipto em solução nutritiva. O trabalho foi realizado de novembro de 2008 a janeiro de 2009, em casa de vegetação. Foi utilizado delineamento inteiramente casualizado em esquema fatorial fracionado $(4 \times 4 \times 4)^{1 / 2}$, perfazendo 32 tratamentos com três repetições. Os tratamentos se constituíram de quatro doses dos nutrientes de $\mathrm{N}\left(50 ; 100 ; 200\right.$ e $\left.400 \mathrm{mg} \mathrm{L}^{-1}\right)$ na forma de ureia, $\mathrm{P}\left(7,5 ; 15 ; 30\right.$ e $\left.60 \mathrm{mg} \mathrm{L}^{-1}\right)$ na forma de ácido fosfórico e $\mathrm{K}\left(50 ; 100 ; 200\right.$ e $\left.400 \mathrm{mg} \mathrm{L}^{-1}\right)$ na forma de cloreto de potássio em solução nutritiva. Houve efeito significativo apenas para as doses de $\mathrm{N}$ isoladamente, para a biomassa seca das miniestacas e biomassa seca da parte aérea e raiz das minicepas com efeito linear decrescente, com o aumento das doses de $\mathrm{N}$. A dose de $50 \mathrm{mg} \mathrm{L}^{-1}$ de $\mathrm{N}$ proporcionou maior acúmulo de biomassa seca das miniestacas e minicepas. A distribuição percentual da biomassa seca e dos nutrientes na biomassa seca das miniestacas, parte aérea e raízes das minicepas variou em relação às doses de $\mathrm{N}$. A extração de macronutrientes pelas miniestacas de $6 \mathrm{~cm}$ na dose de $50 \mathrm{mg} \mathrm{L}^{-1}$ de $\mathrm{N}$ seguiu a seguinte ordem decrescente de $\mathrm{N}>\mathrm{S}>\mathrm{P}>\mathrm{K}>\mathrm{Ca}>\mathrm{Mg}$ e micronutrientes de $\mathrm{Cu}>\mathrm{B}>\mathrm{Mn}>\mathrm{Fe}>\mathrm{Zn}$.
\end{abstract}

Palavras-chave: Eucalyptus urophylla; fertilização; minijardim clonal.

\footnotetext{
ABSTRACT

The nutritional status of the matrix plant has a great importance for the maintenance of its vegetative vigor, which determines the sprouts production and also the rooting percentage and the speed of root formation, since the macro and micronutrients are involved in biochemical and physiological vital processes for the plant. Thus, this study aimed to evaluate the influence of NPK fertilizer on dry biomass production by minicuttings and mini-stumps and the percentage distribution of nutrients in a simple hybrid plant of Eucalyptus

1. Engenheiro Florestal, Msc., Rua da Glória, 187, CEP 39100-000, Diamantina (MG). joseeflorestal@yahoo.com.br

2. Engenheiro Agrônomo, Dr., Professor Associado do Departamento de Agronomia, Universidade Federal dos Vales do Jequitinhonha e Mucuri, Rua da Glória, 187, CEP 39100-000, Diamantina (MG). Bolsista do CNPq. ebsilva@ufvjm.edu.br

3. Engenheiro Florestal, Dr., Professor Adjunto do Departamento de Engenharia Florestal, Universidade Federal dos Vales do Jequitinhonha e Mucuri, Rua da Glória, 187, CEP 39100-000, Diamantina (MG). Bolsista do CNPq. silviculturaufvjm@yahoo.com.br

4. Engenheiro Agrônomo, Dr., Professor Adjunto do Departamento de Engenharia Florestal, Universidade Federal dos Vales do Jequitinhonha e Mucuri, Rua da Glória, 187, CEP 39100-000, Diamantina (MG). grazziotti@yahoo.com.br

5. Engenheiro Agrônomo, Dr., Professor Associado do Departamento de Agronomia, Universidade Federal dos Vales do Jequitinhonha e Mucuri, Rua da Glória, 187, CEP 39100-000, Diamantina (MG). cunha.fernandes@ufvjm.edu.br

6. Engenheiro Agrônomo, Msc., Rua da Glória, 187, CEP 39100-000, Diamantina (MG). juanagronomia@hotmail. com/agro.cassio@hotmail.com

Recebido para publicação em 30/03/2010 e aceito em 29/06/2011
}

Ci. Fl., v. 22, n. 3, jul.-set., 2012 
urophylla in nutrient solution. The experiment was realized from November, 2008 to January, 2009 in a vegetation environment. The experimental design was a completely randomized in factorial scheme with a fractional factorial $(4 \times 4 \times 4)^{1 / 2}$, adding up to 32 treatments with three replicates. The treatments consisted of four levels nutrients of nitrogen $\left(50,100,200\right.$ and $\left.400 \mathrm{mg} \mathrm{L}^{-1}\right)$ in the urea form, P $(7.5,15,30$ and $60 \mathrm{mg}$ $\left.\mathrm{L}^{-1}\right)$ in the phosphoric acid form and $\mathrm{K}\left(50,100,200\right.$ and $\left.400 \mathrm{mg} \mathrm{L}^{-1}\right)$ in the potassium chloride form, in the nutrient solution. There was significant effect only to the $\mathrm{N}$ doses to the dry mass of mini-cuttings and the dry mass of shoots and roots of mini-stumps with decreasing linear effect against $\mathrm{N}$ doses increasing. The doses of $50 \mathrm{mg} . \mathrm{L}^{-1}$ of $\mathrm{N}$ promoted higher accumulation of dry mass in mini-cuttings and mini-stumps. The percentage distribution of dry mass and of the nutrients in dry mass of mini-stumps, shoots and roots of mini-stumps varied in relation to $\mathrm{N}$ doses. Macronutrients extraction from mini-stumps of $6 \mathrm{~cm}$ at a dose of $50 \mathrm{mg} \mathrm{L}^{-1}$ of $\mathrm{N}$ followed the order of decreasing $\mathrm{N}>\mathrm{S}>\mathrm{P}>\mathrm{K}>\mathrm{Ca}>\mathrm{Mg}$ and micronutrients $\mathrm{Cu}>\mathrm{B}>$ $\mathrm{Mn}>\mathrm{Fe}>\mathrm{Zn}$.

Keywords: Eucalyptus urophylla; fertilization; mini-clonal garden.

\section{INTRODUÇÃO}

A propagação vegetativa é uma realidade presente nas empresas florestais que optaram pela silvicultura clonal do eucalipto (CUNHA et al., 2005). O atual estágio de produção de mudas de eucalipto criou um sistema conhecido como minijardim clonal, em que as minicepas ou microcepas podem ser conduzidas por sistema hidropônico, utilizando-se diferentes substratos para fixação (WENDLING et al., 2000).

A nutrição mineral utilizada no minijardim clonal é de fundamental importância para a propagação vegetativa, a qual é composta por macro e micronutrientes, e deveria ser variável de acordo com o material genético (clone), ambiente e sistema de condução das mini ou microcepas. Esses fatores determinam a melhor formulação e balanço dos elementos que compõem a solução nutritiva (ASSIS et al., 2004) e a sua resistência a pragas e doenças (SILVEIRA e HIGASHI, 2003).

O estado nutricional da planta-matriz é de grande importância, não apenas quanto ao aspecto do vigor vegetativo e da produção de brotos, mas também em razão de os elementos minerais apresentarem, nas estacas, efeito altamente significativo nos índices de enraizamento e na velocidade de formação das raízes, uma vez que macro e micronutrientes estão envolvidos nos processos bioquímicos e fisiológicos vitais à planta (ASSIS et al., 2004).

Portanto, as informações básicas sobre a nutrição mineral assumem grande importância no processo de propagação vegetativa. Entretanto, os padrões ideais das concentrações de nutrientes não estão ainda bem estabelecidos (ALFENAS et al., 2004). Higashi et al. (2005) determinaram faixas adequadas de macro e micronutrientes para o processo de miniestaquia, mas que, segundo Cunha et al. (2008), não se enquadram para diferentes materiais genéticos.

Assim, o objetivo deste trabalho foi avaliar a influência da adubação NPK sobre a partição de biomassa seca e nutriente em minicepas de eucalipto em solução nutritiva.

\section{MATERIAL E MÉTODOS}

O trabalho foi realizado de novembro de 2008 a fevereiro de 2009 , em casa de vegetação do Departamento de Agronomia da Faculdade de Ciências Agrárias da Universidade Federal dos Vales do Jequitinhonha e Mucuri (UFVJM), no município de Diamantina/MG, coordenadas de $18^{\circ} 15^{\prime}$ de latitude sul e $43^{\circ} 36^{\prime}$ de longitude Oeste e altitude $1.400 \mathrm{~m}$. Durante o experimento na casa de vegetação a temperatura e umidade relativas médias foram, respectivamente, de $24,3{ }^{\circ} \mathrm{C}$ e $68,4 \%$.

$\mathrm{O}$ experimento foi conduzido em sistema hidropônico com aeração por ar comprimido, utilizando mudas de um híbrido simples de Eucalyptus urophylla, com 45 dias de idade, provenientes da empresa ArcelorMittal BioEnergia de Itamarandiba/MG, que foram selecionadas com altura de $15,0 \pm 0,1 \mathrm{~cm}$ e o diâmetro do coleto de $3,0 \pm 0,5 \mathrm{~mm}$.

Foi utilizado um delineamento inteiramente casualizado em esquema fatorial fracionado $(4 \times 4 \times 4)^{1 / 2}$, perfazendo 32 tratamentos com três repetições, totalizando 96 parcelas experimentais com seis minicepas cada, espaçadas em $10 \mathrm{x}$ $10 \mathrm{~cm}$. Nos arranjos do tipo fatorial, todas as combinações dos tratamentos são avaliadas. Quando esse delineamento é fracionado, algumas 
dessas combinações são eliminadas, de acordo com tabelas estatísticas, que variam com o experimento (CONAGIN et al., 1997). Os tratamentos se constituíram de quatro doses dos nutrientes: $\mathrm{N}(50$, 100,200 e $\left.400 \mathrm{mg} \mathrm{L}^{-1}\right)$ na forma de ureia, $\mathrm{P}(7,5$, 15,30 e $\left.60 \mathrm{mg} \mathrm{L}^{-1}\right)$ na forma de ácido fosfórico e $\mathrm{K}$ $\left(50,100,200\right.$ e $\left.400 \mathrm{mg} \mathrm{L}^{-1}\right)$ na forma de cloreto de potássio.

As concentrações dos outros nutrientes da solução nutritiva foram: $100 \mathrm{mg} \mathrm{Ca} ; 25 \mathrm{mg} \mathrm{Mg} ; 35$ mg S; 0,3 mg B; 0,03 mg Cu; 0,3 mg Fe; 0,3 mg Mn; 0,05 mg Zn e 0,01 mg Mo por litro de solução nutritiva (HIGASHI et al., 2005). Para facilitar a preparação da solução nutritiva e aplicação dos tratamentos, utilizou-se solução estoque preparada com os seguintes reagentes p.a.: ureia, ácido fosfórico, cloreto de potássio, sulfato de cálcio, cloreto de cálcio, sulfato de magnésio, cloreto de magnésio, ácido bórico, sulfato de cobre, sulfato de manganês, sulfato de zinco, molibdato de sódio e cloreto ferroso + EDTA. Durante todo o experimento utilizou-se água deionizada para preparação da solução nutritiva e o $\mathrm{pH}$ foi ajustado para 5,4 \pm 0,3.

$\mathrm{Na}$ instalação do experimento, as mudas tiveram seu sistema radicular lavado em água corrente para a retirada do substrato. Posteriormente, foram fixadas em placas de isopor de $2,0 \mathrm{~cm}$ de espessura e colocadas em bacias com capacidade para 10 litros de solução nutritiva.

Inicialmente, as mudas passaram por um período de 12 dias de aclimatação na solução nutritiva, quando a concentração dos nutrientes foi aumentada gradualmente $(10,25,50$ e 75 $\%$ da concentração dos nutrientes), a cada três dias, até chegar a $100 \%$ da menor dose de NPK (respectivamente $50,7,5$ e $50 \mathrm{mg} \mathrm{L}^{-1}$ ) e total dos outros nutrientes. Nessa fase, o $\mathrm{pH}$ foi ajustado apenas no dia da troca da solução. Com as mudas aclimatadas, a solução nutritiva passou a ser trocada a cada sete dias e o $\mathrm{pH}$ foi ajustado três vezes a cada intervalo de troca, ao longo de todo o experimento.

Aos 21 dias após a instalação (DAI) do experimento, com as mudas adaptadas à solução nutritiva, o ápice das mudas foi cortado, deixandose pelo menos dois pares de folhas completamente formadas, visando à formação das minicepas. Durante um período de 40 dias, as minicepas passaram por quatro desbastes para a formação da taça para, então, serem aplicados os tratamentos.

A aplicação dos tratamentos foi realizada aos 61 DAI e foi dividida em duas partes para proporcionar a aclimatação das minicepas aos tratamentos com doses mais elevadas. Assim, no primeiro momento, aos $61 \mathrm{DAI}$, foi aplicada a metade das doses mais altas, 400, 60 e $400 \mathrm{mg}$ $\mathrm{L}^{-1}$, respectivamente, de NPK, e total das outras doses de NPK. Aos 68 DAI, os tratamentos foram completamente aplicados.

Foram efetuadas quatro coletas de miniestacas apicais durante um período de 30 dias, sendo a primeira coleta realizada aos 75 DAI. As miniestacas foram coletadas com $6,0 \mathrm{~cm}$ de comprimento e um par de folhas completamente formado. Utilizou-se um gabarito de $6 \mathrm{~cm}$ para garantir que as miniestacas fossem todas coletadas com o mesmo tamanho. Após a última coleta de miniestaca, foi coletada uma minicepa por parcela experimental.

As miniestacas coletadas foram contadas, lavadas em água deionizada, armazenadas em saco de papel por unidade experimental a cada coleta, secas em estufa de circulação forçada a $65{ }^{\circ} \mathrm{C}$ até peso constante e pesadas. As minicepas lavadas foram armazenadas em saco de papel por unidade experimental separando raiz e parte aérea, secas em estufa de circulação forçada a $65{ }^{\circ} \mathrm{C}$ até peso constante e pesadas. A determinação dos teores dos macronutrientes $(\mathrm{N}, \mathrm{P}, \mathrm{K}, \mathrm{Ca}, \mathrm{Mg}$ e $\mathrm{S})$ e dos micronutrientes $(\mathrm{B}, \mathrm{Cu}, \mathrm{Fe}, \mathrm{Mn}$ e $\mathrm{Zn})$ na biomassa seca das miniestacas e minicepas (parte aérea e raízes) foi realizada conforme metodologia descrita por Malavolta et al. (1997).

As variáveis utilizadas para verificar o efeito dos tratamentos foram: biomassa seca da parte aérea por minicepa (PAMC), biomassa seca das raízes por minicepa (RMC), biomassa seca acumulada das miniestacas (ME), onde se avaliou a distribuição percentual da biomassa seca e dos nutrientes em cada parte.

Os dados foram submetidos à análise de variância e estudo de regressão para as doses de N, P e K, utilizando-se, respectivamente, o PROC GLM e PROC REG do software SAS para Windows. As médias dos percentuais de distribuição de nutrientes na biomassa seca de minicepa e miniestacas foram submetidas ao teste de Scott \& Knott, a 5 $\%$ de probabilidade. Os coeficientes angulares das regressões lineares ajustadas foram submetidos ao teste de T a $1 \%$ de probabilidade.

\section{RESULTADOS E DISCUSSÃO}

Houve efeito significativo isoladamente para as doses de $\mathrm{N}$ sobre biomassa seca da parte 
aérea por minicepa (PAMC), biomassa seca das raízes por minicepa (RMC) e biomassa seca das miniestacas (ME) (Tabela 1), com efeito linear decrescente (Figura 1). Logo, para o material genético em estudo, as doses de $50 \mathrm{mg} \mathrm{L}^{-1}$ de $\mathrm{N}$ foram as que proporcionaram maior produção de biomassa seca.

$\mathrm{O}$ aumento das doses de $\mathrm{N}$ de 50 para 400 mg L ${ }^{-1}$ ocasionou redução de 16,$0 ; 9,8$ e 47,2 \% para, respectivamente, PAMC, RMC e ME, sendo mais acentuada na $\mathrm{ME}$ em relação às demais variáveis, como pode ser comprovado pelos coeficientes angulares das regressões lineares dessas variáveis, que seguiram a mesma tendência de queda quando comparados (Figura 1). A maior redução na $\mathrm{ME}$ com aumento das doses de $\mathrm{N}$ deve-se ao fato do material ser coletado e necessitar de $\mathrm{N}$ para crescer novamente. A absorção de $\mathrm{N}$ pelo eucalipto é preferencialmente na forma de amônio (GRESPAN et al., 1998) atribuído à adaptação evolutiva da espécie a solos ácidos. A utilização da ureia como fonte de $\mathrm{N}$, que apresenta nitrificação mais intensa quando comparada a outras fontes (SILVA e VALE, 2000), com aumentos lineares na taxa de nitrificação com o acréscimo de $\mathrm{pH}$ de 5,0 a 8,0 (KYVERYGA et al., 2004). Como o pH da solução foi corrigido para $5,4 \pm 0,3$, ocorreu o favorecimento da nitrificação do amônio proveniente da ureia e aumento dos níveis de nitrato em solução com o aumento das doses de $\mathrm{N}$.

A distribuição percentual da biomassa seca em relação às doses de $\mathrm{N}$ mostrou diferença no acúmulo de biomassa seca para as variáveis estudadas (Figura 2). A PAMC e a RMC apresentaram aumento, com maior acúmulo percentual de biomassa seca de, respectivamente, 29 e $54 \%$ na dose de $400 \mathrm{mg} \mathrm{L}^{-1}$ (Figura 2). Já para a ME, a distribuição percentual da biomassa seca reduziu com o aumento das doses de N, sendo que o maior acúmulo percentual de biomassa seca (29 \%) ocorreu na dose de $50 \mathrm{mg} \mathrm{L}^{-1}$ (Figura 2). A adubação nitrogenada na forma de ureia afetou mais o acúmulo de biomassa seca da miniestaca do que da minicepa. Portanto, a adubação nitrogenada deve ser ajustada de acordo com o objetivo da produção de miniestaquia de eucalipto.

Ao se estimar valores de PAMC e RMC para as doses de $\mathrm{N}$, a partir das equações geradas (Figura 1), e calculando-se a razão parte aérea/ raiz, esta razão aumentou de 3,0 , na dose de $50 \mathrm{mg}$ $\mathrm{L}^{-1}$, para 4,0 , na dose de $400 \mathrm{mg} \mathrm{L}^{-1}$. Esse aumento ocorreu porque altos níveis de nutrientes favorecem o desenvolvimento da parte aérea em relação à raiz (BANGERTH et al., 2000).

Houve diferença significativa pelo teste de médias paraa porcentagem demacroemicronutrientes por componente vegetal em função das doses de $\mathrm{N}$ (Figuras 3 e 4). De modo geral, o aumento das doses de $\mathrm{N}$ ocasionou a redução da quantidade de macro e micronutrientes na ME, e para a PAMC e RMC o comportamento foi variado, mas seguindo uma tendência da dose de $400 \mathrm{mg} \mathrm{L}^{-1}$ acumular maior ou menor quantidade de macro e micronutrientes em relação às três menores doses, que tenderam a ser semelhantes no acúmulo de nutrientes (Figuras $3 \mathrm{e}$ 4). A maior parte dos nutrientes, com exceção do $\mathrm{Cu}$

TABELA 1: Análise de variância de biomassa seca de miniestacas (ME) de parte aérea de minicepa (PAMC), de raízes de minicepa (RMC) em função das doses de NPK.

TABLE 1: Variance analysis of dry biomass of mini-cuttings (ME), shoots of mini-stumps (PAMC) and roots of mini-stumps (RMC) in function of doses of NPK.

\begin{tabular}{lcccc}
\hline Fator de variação & GL & \multicolumn{3}{c}{ Quadrado médio } \\
\cline { 3 - 5 } & & ME & PAMC & RMC \\
\hline $\mathrm{N}$ & 3 & $1,87^{* *}$ & $1,15^{* *}$ & $0,21^{* *}$ \\
$\mathrm{P}$ & 3 & 0,05 & 0,04 & 0,03 \\
$\mathrm{~K}$ & 3 & 0,05 & 0,03 & 0,03 \\
$\mathrm{~N}^{*} \mathrm{P}$ & 9 & 0,04 & 0,17 & 0,03 \\
$\mathrm{~N}^{*} \mathrm{~K}$ & 7 & 0,05 & 0,34 & 0,08 \\
$\mathrm{P}^{*} \mathrm{~K}$ & 3 & 0,02 & 0,24 & 0,07 \\
Erro & 67 & 0,08 & 0,32 & 0,06 \\
\hline Média geral & & 1,11 & 2,52 & 1,27 \\
\hline CV $(\%)$ & & 26,10 & 22,44 & 20,07 \\
\hline
\end{tabular}

Em que: $* *=$ significativo a $1 \%$ pelo teste de $\mathrm{F}$. 
e Fe, foi acumulada na MSPAMC e MSME (Figuras 3 e 4). Juntas, apresentaram valores médios de: $93 \%$ do N, $81 \%$ do P, $84 \%$ do K, $76 \%$ do Ca, $74 \%$ do $\mathrm{Mg}, 91 \%$ do S, $69 \%$ do B, $22 \%$ do $\mathrm{Cu}, 24 \%$ do Fe, $92 \%$ do Mn e $66 \%$ do $\mathrm{Zn}$.

A maior parte do $\mathrm{N}$ e do $\mathrm{S}$, respectivamente, absorvida em média de 82 e $81 \%$ foi direcionada para a MSME (Figura 3). Isso porque as assimilações de $\mathrm{N}$ e $\mathrm{S}$ são bem coordenadas, com a deficiência de um elemento reprimindo a via assimilatória do outro (KOPRIVOVA et al., 2000). A regulação ocorre não apenas em nível de proteínas, dada a exigência de $\mathrm{N}$ e $\mathrm{S}$ nos aminoácidos, mas também na transcrição dos mRNAs codificando para as enzimas envolvidas na assimilação desses dois macronutrientes (KOPRIVA et al., 2004). Durante o rápido crescimento vegetativo, que é o caso das miniestacas, são altas as taxas de redução de $\mathrm{N}$ e $\mathrm{S}$, e síntese de aminoácidos, que são utilizados para a síntese de clorofila, rubisco e outras proteínas. Com isso, é baixo o nível de aminoácidos no floema que entra nas raízes (IMSANDE e TOURAINE, 1994).

$\mathrm{O} \mathrm{P}$ e o $\mathrm{K}$ acumularam-se principalmente na ME e PAMC, mas com comportamento inverso com o aumento das doses de N (Figura 3), que pode ser explicado pela atuação desses nutrientes. O fosfato é um elemento transferidor de energia, importante no metabolismo e no desenvolvimento vegetal (EPSTEIN e BLOOM, 2006). Já o K atua na síntese de proteínas, ativação de mais de 50 enzimas, manutenção do turgor celular, regulação da abertura e fechamento estomático, transporte e o armazenamento de carboidratos, síntese de amido nas folhas, entre outras (MALAVOLTA, 2006; MEURER, 2006; TAIZ e ZEIGER, 2008). Isso explica a maior porcentagem de $\mathrm{P}$ e $\mathrm{K}$ na ME e PAMC, regiões de intenso crescimento e fotossinteticamente ativas.

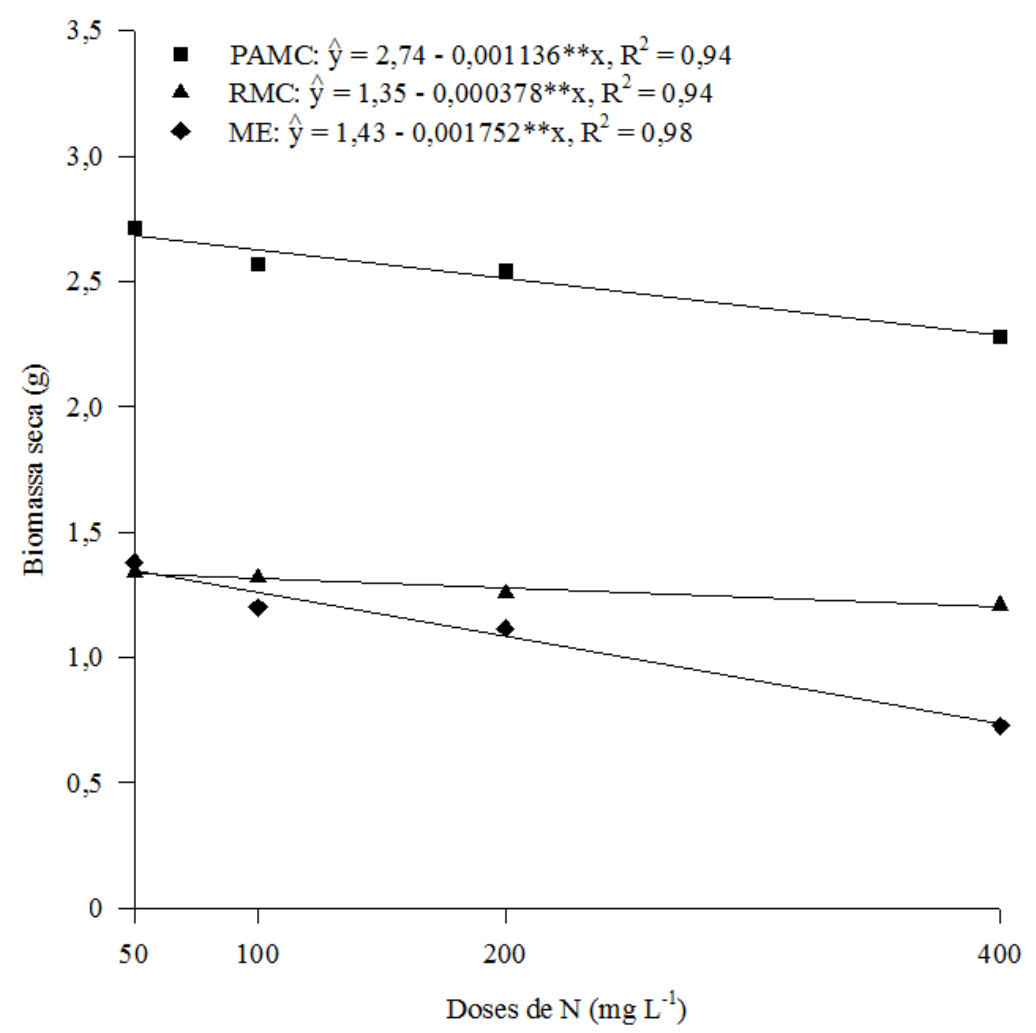

FIGURA 1: Relação entre a biomassa seca de miniestacas média por minicepas (ME), de parte aérea de minicepa (PAMC) e de raízes de minicepa (RMC) de um híbrido simples de Eucalyptus urophylla, em função de doses de $\mathrm{N}$ em solução nutritiva num período de 30 dias (** significativo a $1 \%$ pelo teste de T).

FIGURE 1: Relationship between the dry mass of mini-cuttings per mini-stumps mean (ME), the shoot mini-stumps (PAMC) and the roots of mini-stumps (RMC) of a simple hybrid of Eucalyptus urophylla in function of $\mathrm{N}$ in nutrient solution over a period of 30 days (** significant at $1 \%$ by t test). 
Para a distribuição do $\mathrm{Ca}$ e $\mathrm{Mg}$, o maior acúmulo percentual ocorreu na PAMC (Figura 3). O maior acúmulo de Ca na PAMC se justifica por ser o macronutriente mais proeminente no apoplasto, atuando na interligação das cadeias pécticas (MATOH e KOBAYASHI, 1998), além de ser essencial para manter a integridade estrutural e funcional das membranas e da parede celular (BANGERTH, 1979). A distribuição do Mg, assim como o de $\mathrm{Ca}$, apresentou maior porcentagem média de acúmulo na PAMC, de $44 \%$ (Figura 3). O $\mathrm{Mg}$ ativa mais enzimas que qualquer outro elemento na planta, sendo a sua principal atuação na ativação da ATPase, que constitui uma família de enzimas que catalisam a hidrólise do ATP para originar ADP e fosfato inorgânico, tendo como elemento constituinte principal o P (BRISKIN e POOLE, 1983). Assim, como as miniestacas são tecidos em desenvolvimento, portando-se como drenos, a parte aérea (PAMC) é a fonte de energia para o crescimento e desenvolvimento das minicepas. Por isso, há maior presença de $\mathrm{Mg}$ para a manutenção do metabolismo da planta.

O maior acúmulo percentual médio de
B deu-se na PAMC, seguido pela RMC e ME, com respectivamente 47, 31 e $22 \%$ (Figura 4). A distribuição percentual do $\mathrm{B}$ em cada variável segue o acúmulo médio de biomassa seca da minicepa e da miniestaca. Isto se deve ao papel desempenhado pelo B no metabolismo dos carboidratos, transporte de açúcares através das membranas, na síntese de ácidos nucleicos e de fito-hormônios, na formação de paredes celulares e divisão celular (BLEVINS e LUKASZEWSKI, 1998; BROWN et al., 2002). Em decorrência do papel do B citado acima, observouse a sua importância na miniestaquia, uma vez que, tanto o conteúdo de $\mathrm{B}$ quanto a biomassa seca das miniestacas, diminuíram com o aumento das doses de N.

Houve maior acúmulo de $\mathrm{Cu}$ e o $\mathrm{Fe}$ na RMC, de, respectivamente, 78 e $76 \%$ (Figura 4). Isso ocorreu em razão da capacidade de troca de cátions da raiz. Concentrações mais elevadas de micronutrientes catiônicos podem ser encontradas nas raízes, em comparação à parte aérea, simplesmente como resultado das ligações preferenciais no apoplasto das células radiculares (YRUELA, 2005).

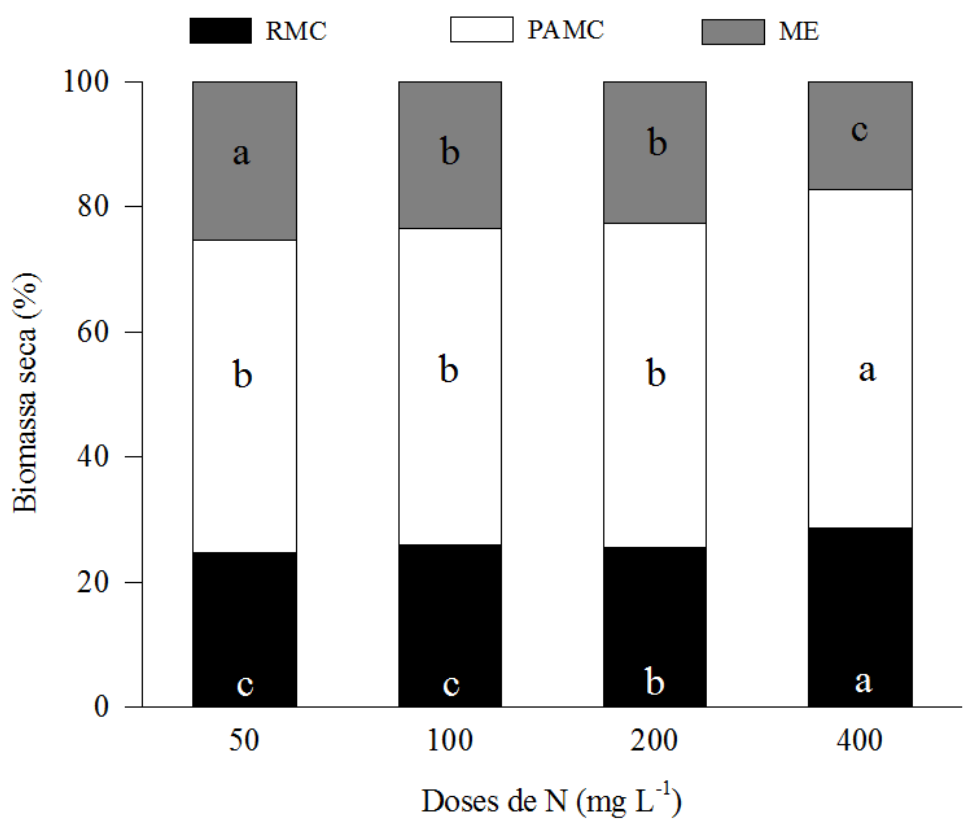

FIGURA 2: Distribuição percentual de biomassa seca de raízes de minicepa (RMC), parte aérea de minicepa (PAMC) e miniestacas por minicepas (ME) de um híbrido simples de Eucalyptus urophylla, em função de doses de $\mathrm{N}$ em solução nutritiva num período de 30 dias. Médias seguidas pela mesma letra, para cada biomassa seca, não diferem entre si pelo teste Scott \& Knott a $5 \%$.

FIGURE 2: Percentage distribution dry mass of roots mini-stumps (RMC), shoots mini-stumps (PAMC) and mini-cuttings for mini-stumps (ME) for a simple hybrid of Eucalyptus urophylla in function of $\mathrm{N}$ in nutrient solution over a period of 30 days. Means followed by the same letter for each dry biomass, do not differ by Scott \& Knott test at $5 \%$ 


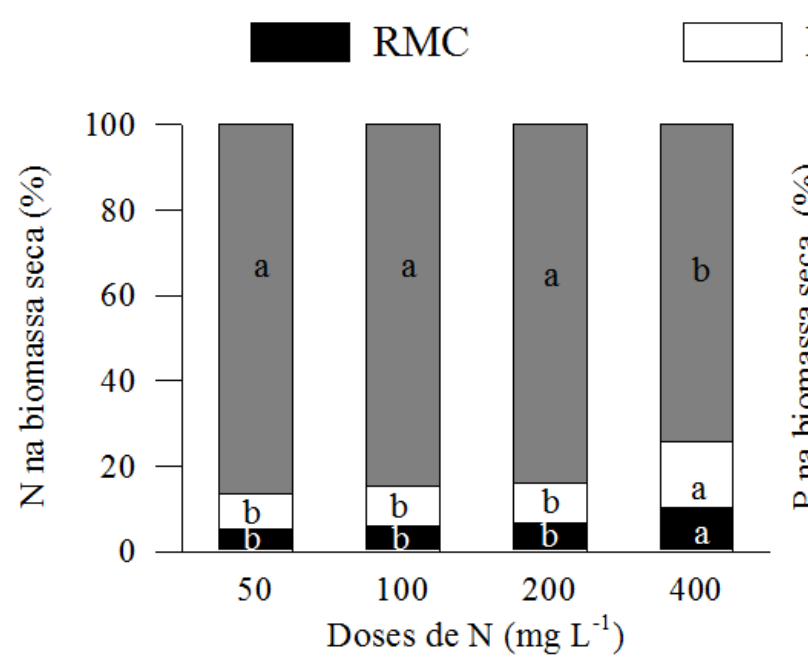

PAMC $\square$ ME
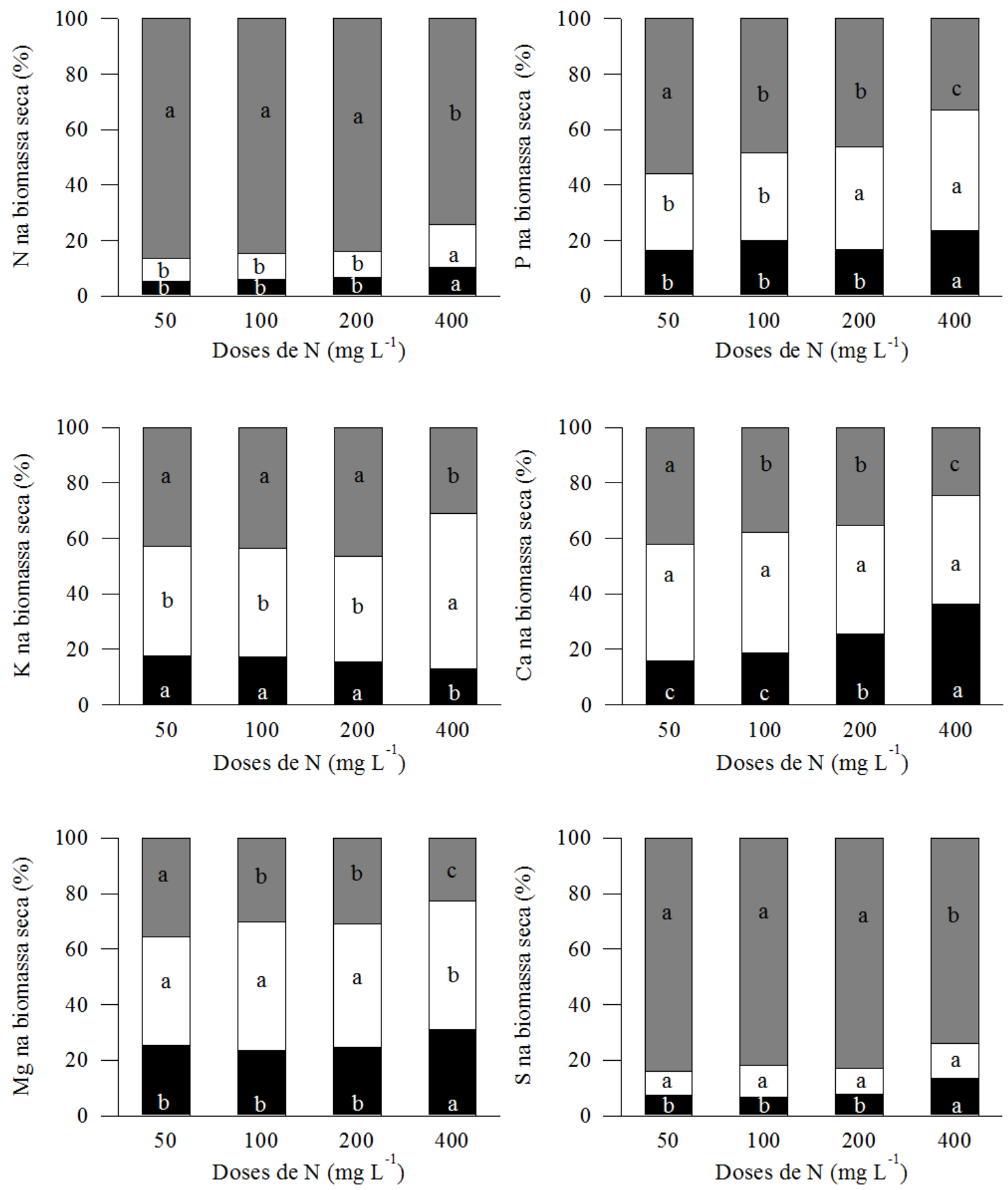

FIGURA 3: Distribuição percentual de acúmulo de macronutrientes na biomassa seca de raízes de minicepa (RMC), parte aérea de minicepa (PAMC) e miniestacas por minicepas (ME) de um híbrido simples de Eucalyptus urophylla, em função de doses de $\mathrm{N}$ em solução nutritiva num período de 30 dias. Médias seguidas pela mesma letra, para cada biomassa seca, não diferem entre si pelo teste Scott \& Knott a $5 \%$.

FIGURE 3: Distribution of macronutrients accumulation in the root dry mass of mini-stumps (RMC), shoots mini-stumps (PAMC) and mini-cuttings for mini-stumps (ME) for a simple hybrid of Eucalyptus urophylla in function of $\mathrm{N}$ in nutrient solution over a period of 30 days. Averages followed by the same letter for each dry biomass, do not differ by Scott \& Knott test at $5 \%$. 

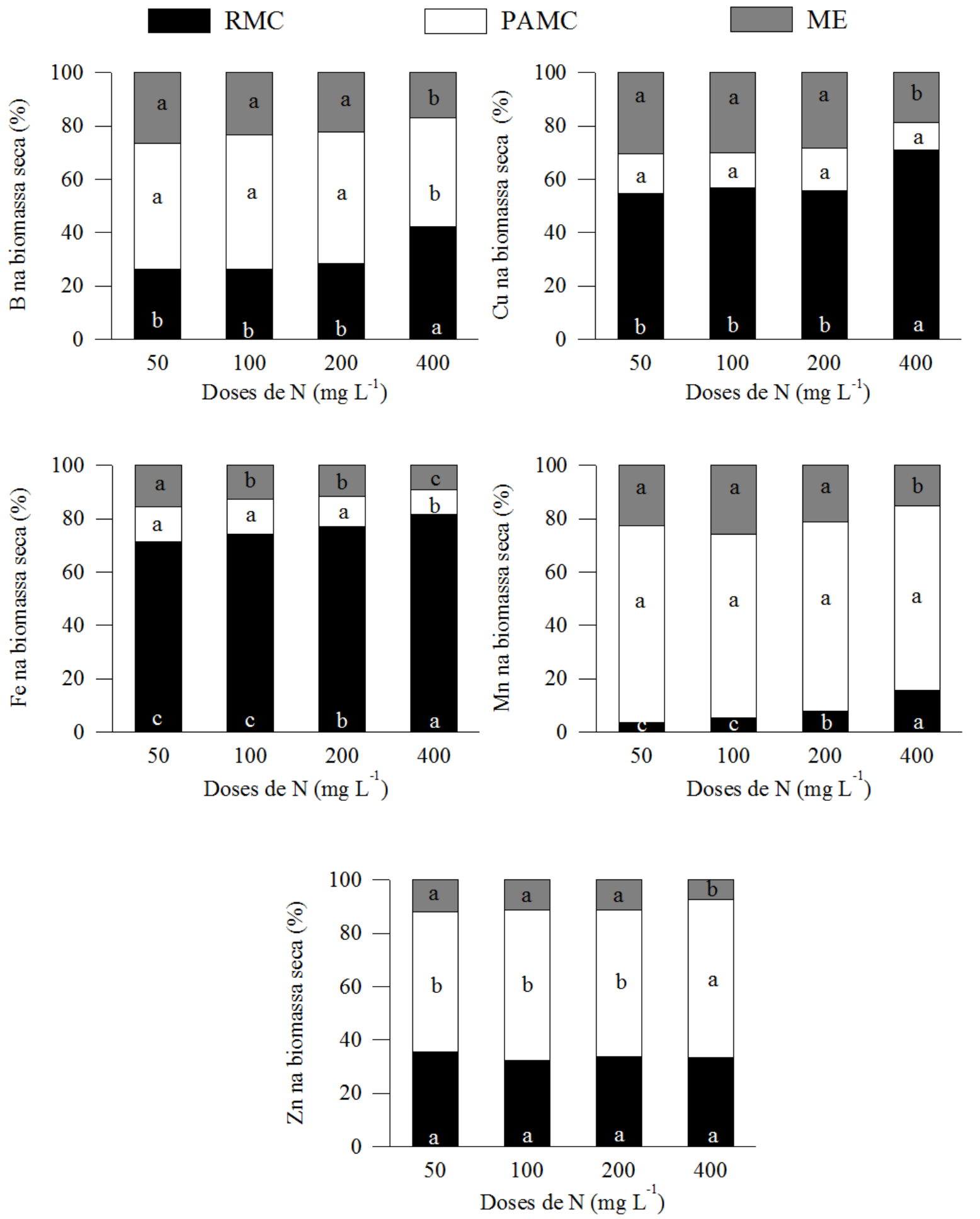

FIGURA 4: Distribuição percentual de acúmulo de micronutrientes na biomassa seca de raízes de minicepa (RMC), parte aérea de minicepa (PAMC) e miniestacas por minicepas (ME) de um híbrido simples de Eucalyptus urophylla, em função de doses de $\mathrm{N}$ em solução nutritiva num período de 30 dias. Médias seguidas pela mesma letra, para cada biomassa seca, não diferem entre si pelo teste Scott \& Knott a $5 \%$.

FIGURE 4: Distribution of micronutrients accumulation in the root dry mass of mini-stumps (RMC), shoots mini-stumps (PAMC) and mini-cuttings for mini-stumps (ME) for a simple hybrid of Eucalyptus urophylla in function of $\mathrm{N}$ in nutrient solution over a period of 30 days. Means followed by the same letter for each dry biomass do not differ by Scott \& Knott test at $5 \%$. 
O Mn foi o nutriente que apresentou, em média, o maior acúmulo na PAMC, de $72 \%$, seguido pelo o $\mathrm{Zn}$, de $56 \%$. As funções do Mn e do $\mathrm{Zn}$ estão diretamente ligadas ao metabolismo das plantas. Entre suas funções, o Mn, por exemplo, participa junto com o $\mathrm{Cl}$ na evolução do oxigênio no processo fotossintético (HAKALA et al., 2005); já o Zn participa do metabolismo do N (HAMLIN e BARKER, 2006, BROADLEY et al., 2007). Assim, a grande porcentagem de Mn e $\mathrm{Zn}$ na PAMC se deve ao fato de a parte aérea ser responsável por fornecer energia pela fotossíntese e proteínas para o crescimento e desenvolvimento da planta, uma vez que as miniestacas se comportam como drenos.

No maior acúmulo de biomassa seca na minicepa e miniestaca na dose de $\mathrm{N}$ de $50 \mathrm{mg} \mathrm{L}^{-1}$ (Figura 2), a ordem decrescente de acúmulo de macronutrientes foi a seguinte: $\mathrm{Mg}>\mathrm{K}>\mathrm{P}>\mathrm{Ca}>$ $\mathrm{S}>\mathrm{N}$ na RMC; $\mathrm{Ca}>\mathrm{K}>\mathrm{Mg}>\mathrm{P}>\mathrm{S}>\mathrm{N}$ na PAMC e $\mathrm{N}>\mathrm{S}>\mathrm{P}>\mathrm{K}>\mathrm{Ca}>\mathrm{Mg}$ na ME (Figura 3). Da mesma forma, para o acúmulo dos micronutrientes seguiu a tendência: $\mathrm{Fe}>\mathrm{Cu}>\mathrm{Zn}>\mathrm{B}>\mathrm{Mn}$ na $\mathrm{RMC}$; $\mathrm{Mn}>\mathrm{Zn}>\mathrm{B}>\mathrm{Cu}>\mathrm{Fe}$ na PAMC e $\mathrm{Cu}>\mathrm{B}>\mathrm{Mn}>$ $\mathrm{Fe}>\mathrm{Zn}$ na ME (Figura 4). A variação na ordem de acúmulo de macro e micronutrientes está associada à função que eles desempenham no metabolismo da planta, de acordo com a taxa de crescimento, órgão vegetal (raiz, parte aérea e miniestaca) e ontogenia da planta.

A variação da distribuição percentual dos nutrientes e da biomassa seca na ME, PAMC e RMC em função das doses de $\mathrm{N}$ mostra a importância do cuidado na hora da amostragem das minicepas para análise foliar. As amostras devem ser compostas de miniestacas, pois o seu teor de nutrientes foliar irá representar melhor o efeito da dose do nutriente aplicada sobre a produção de miniestacas.

\section{CONCLUSÕES}

$\mathrm{O}$ aumento da dose de $\mathrm{N}$ promoveu redução na biomassa seca das miniestacas e da parte aérea e raízes das minicepas.

A maior produção de biomassa seca de miniestaca e minicepa foi sob a dose de $50 \mathrm{mg} \mathrm{L}^{-1}$ para nitrogênio e não houve resposta das doses de $\mathrm{P}$ e K.

A distribuição percentual da biomassa seca e dos nutrientes variou em função do aumento das doses de $\mathrm{N}$ e do componente vegetal, miniestaca, parte aérea e raiz das minicepas.
A extração de macronutrientes pelas miniestacas de $6 \mathrm{~cm}$ na dose de $50 \mathrm{mg} \mathrm{L}^{-1}$ de $\mathrm{N}$ seguiu a seguinte ordem decrescente de $\mathrm{N}>\mathrm{S}>\mathrm{P}$ $>\mathrm{K}>\mathrm{Ca}>\mathrm{Mg}$ e micronutrientes de $\mathrm{Cu}>\mathrm{B}>\mathrm{Mn}$ $>\mathrm{Fe}>\mathrm{Zn}$.

\section{AGRADECIMENTOS}

À Universidade Federal dos Vales do Jequitinhonha e Mucuri (UFVJM), à Coordenação de Aperfeiçoamento de Pessoal de Nível Superior (CAPES) e à Empresa ArcelorMittal BioEnergia, pelo apoio e cooperação.

\section{REFERÊNCIAS BIBLIOGRÁFICAS}

ALFENAS, A. C. et al. Clonagem do eucalipto. In: ALFENAS, A. C. et al. Clonagem e doenças do eucalipto. Viçosa: UFV, 2004. p.58-72.

ASSIS, T.; FETT-NETO, A. G.; ALFENAS, A. C. Current techniques and prospects for the clonal propagation of hardwoods with emphasis on Eucalyptus. In: WALTERS, C.; CARSON, M. (Eds.). Plantation Forest Biotechnology for the $21^{\text {st }}$ Century. India: Research Signpost, 2004. p. 303-333.

BANGERTH, F. C. Calcium-related physiological disorders of plants. Annual Review of Phytopathology, Palo Alto, v. 17, p.97-122, Sept. 1979.

BANGERTH, F. C.; LI, C. J.; GRUBER, J. Mutual interaction of auxin and cytokinins in regulating correlative dominance. Plant Grow Regulation, New York, v. 32, n. 1-2, p. 205-217, Nov. 2000.

BLEVINS, D. G.; LUKASZEWSKI, K. M. Boron in plant structure and function. Annual Review of Plant Physiology and Plant Molecular Biology, Palo Alto, v. 49, p. 481-500, June 1998.

BRISKIN, D. P.; POOLE, R. J. Role of magnesium in the plasma membrane ATPase of red beet. Plant Physiology, Washington, v. 71, n. 4, p. 969-971, Dec. 1983.

BROADLEY, M. R. et al. Zinc in plants. New Phytologist, Lancaster, v. 173, n. 4, p. 677-702, Mar. 2007.

BROWN, P. H. et al. Boron in plant biology. Plant Biology, Freiburg, v. 4, n. 2, p. 205-223, Mar. 2002. CONAGIN,A.; NAGAI, V.; IGUE, T. Delineamento $(1 / 2) 4^{3}$ em blocos de oito unidades. Campinas: Instituto Agronômico de Campinas, 1997. 9 p. (Boletim Científico, 36)

CUNHA, A. C. M. C. M. et al. Relação do 
estado nutricional de minicepas com o número de miniestacas de eucalipto. Scientia Forestalis, Piracicaba, v. 36, n. 79, p. 203-213, set. 2008.

CUNHA, A. C. M. C.; WENDLING, I.; SOUZA JÚNIOR, J. Produtividade e sobrevivência de minicepas de Eucalyptus benthamii Maiden et Cambage em sistema de hidroponia e em tubete. Ciência Florestal, Santa Maria, v.15, n. 3, p. 307310, jul./set. 2005.

EPSTEIN, E.; BLOOM, A. J. Nutrição mineral de plantas: princípios e perspectivas. 2. ed. Londrina: Ed. Planta, 2006. 403 p.

GRESPAN, S. L.; DIAS, L. E.; NOVAIS, L. S. Crescimento e parâmetros cinéticos de absorção de amônio e nitrato por mudas de Eucalyptus spp. submetidas a diferentes relações amônio/nitrato na presença e ausência de fósforo. Revista Brasileira de Ciência do Solo, Viçosa, v. 22, n. 4, p. 667-674, out./dez. 1998.

HAKALA, M. et al. Evidence for the role of the oxygen-evolving manganese complex in photoinhibition of photosystem II. Biochimica et Biophysica Acta Bioenergetics, Amsterdam, v. 1706, n. 1-2, p. 68-90, Jan. 2005.

HAMLIN, R. L.; BARKER, A. V. Influence of ammonium and nitrate nutrition on plant growth and zinc accumulation by indian mustard. Journal of Plant Nutrition, New York, v. 29, n. 8, p. 15231541, Aug. 2006.

HIGASHI, E. N.; SILVEIRA, R. L. V. A.; GONÇALVES, A. N. Monitoramento nutricional e fertilização em macro, mini e microjardim clonal de Eucalyptus. In: GONÇALVES, J. L. M.; BENEDETTI, V., Nutrição e fertilização florestal. Piracicaba: IPEF, 2005. p.192-217.

IMSANDE, J.; TOURAINE, B. N demand and regulation of nitrate uptake. Plant Physiology, Lancaster, v. 105, n. 1, p.3-7, May 1994.

KOPRIVOVA, A. et al. Regulation of sulfate assimilation by nitrogen in Arabidopsis. Plant Physiology, Lancaster , v. 122, n. 3, p. 737-746,
Mar. 2000.

KOPRIVOVA, S. et al. Regulation of sulfate assimilation by nitrogen and sulfur nutrition in poplar trees. Trees Structure and Function, Freiburg, v. 18, n. 3, p. 1432-2285, May 2004.

KYVERYGA, P. M. et al. Soil $\mathrm{pH}$ effects on nitrification of fall-applied anhydrous ammonia. Soil Science Society of American Journal, Madison, v. 68, n. 2, p. 545-551, Mar. 2004.

MALAVOLTA, E. Manual de nutrição mineral de plantas. São Paulo: Ceres, 2006. 638 p.

MALAVOLTA, E.; VITTI, G. C.; OLIVEIRA, S. A. Avaliação do estado nutricional das plantas: princípios e aplicações. 2. ed. Piracicaba: POTAFOS, 1997. 319 p.

MATOH, T.; KOBAYASHI, M. Boron and calcium, essential inorganic constituents of pectic polysaccharides in higher plant cell walls. Journal of Plant Reserach, Tokyo, v. 11, n. 1, p. 179-190, Mar. 1998.

MEURER, E. J. Potássio. In: FERNANDES, M. S. Nutrição mineral de plantas. Viçosa: Sociedade Brasileira de Ciência do Solo, 2006. p.281-298.

SILVA, C. A.; VALE, F. R. Disponibilidade de nitrato em solos brasileiros sob efeito da calagem e de fontes e doses de nitrogênio. Pesquisa Agropecuária Brasileira, Brasília, v. 35, n. 12, p. 2461-2471, dez. 2000.

SILVEIRA, R. L. V. A.; HIGASHI, E. N. Aspectos nutricionais envolvidos na ocorrência de doenças com ênfase para o eucalipto. Piracicaba: IPEF, 2003. 16 p. (Circular Técnica, 2000).

TAIZ, L.; ZEIGER, E. Fisiologia vegetal. 4. ed. Porto Alegre: Artmed, 2008. 820 p.

WENDLING, I. et al. Propagação clonal de híbridos de Eucalyptus spp. por miniestaquia. Revista Árvore, Viçosa, v. 24, n. 2, p. 181-186, mar./abr. 2000.

YRUELA, I. Copper in plants. Brazilian Journal of Plant Physiology, Pelotas, v. 17, n. 1, p. 145156, jan./mar. 2005. 